\title{
Id-1 is not expressed in the luminal epithelial cells of mammary glands
}

\author{
Norihisa Uehara1, Yu-Chien Chou1 ${ }^{1}$, Jose J Galvez², Paola de-Candia ${ }^{3}$, Robert D Cardiff², \\ Robert Benezra ${ }^{3}$ and Gopalan Shyamala ${ }^{1}$
}

1Division of Life Sciences, Lawrence Berkeley National Laboratory, University of California Berkeley, USA
2Department of Pathology, Center for Comparative Medicine, University of California, Davis, USA
'Department of Cell Biology, Memorial Sloan-Kettering Cancer Center, New York, USA

Corresponding author: Gopalan Shyamala (e-mail: Shyamala_Harris@lbl.gov)

Received: 28 June 2002 Revisions received: 7 October 2002 Accepted: 6 November 2002 Published: 9 January 2003

Breast Cancer Res 2003, 5:R25-R29 (DOI 10.1186/bcr560)

(c) 2003 Uehara et al., licensee BioMed Central Ltd (Print ISSN 1465-5411; Online ISSN 1465-542X). This is an Open Access article: verbatim copying and redistribution of this article are permitted in all media for any non-commercial purpose, provided this notice is preserved along with the article's original URL.

\begin{abstract}
Background: The family of inhibitor of differentiation/DNA binding (Id) proteins is known to regulate development in several tissues. One member of this gene family, Id-1, has been implicated in mammary development and carcinogenesis. Mammary glands contain various cell types, among which the luminal epithelial cells are primarily targeted for proliferation, differentiation and carcinogenesis. Therefore, to assess the precise significance of Id-1 in mammary biology and carcinogenesis, we examined its cellular localization in vivo using immunohistochemistry.
\end{abstract}

Methods: Extracts of whole mammary glands from wild type and Id-1 null mutant mice, and tissue sections from paraffinembedded mouse mammary glands from various

Keywords: human, Id-1, immunohistochemistry, mammary glands, mouse developmental stages and normal human breast were subjected to immunoblot and immunohistochemical analyses, respectively. In both these procedures, an anti-ld-1 rabbit polyclonal antibody was used for detection of Id-1.

Results: In immunoblot analyses, using whole mammary gland extracts, Id-1 was detected. In immunohistochemical analyses, however, Id-1 was not detected in the luminal epithelial cells of mammary glands during any stage of development, but it was detected in vascular endothelial cells.

Conclusion: Id-1 is not expressed in the luminal epithelial cells of mammary glands.

\section{Introduction}

Inhibitor of differentiation/DNA binding (Id) proteins belong to a subfamily of helix-loop-helix (HLH) proteins. Four mammalian members of this family (Id1-ld4) have been identified. The distinguishing characteristic of Id proteins is that, unlike the basic HLH proteins, they do not contain a basic DNA binding domain. Nevertheless, they can regulate cell functions primarily by dimerization with other transcriptional regulators, principally basic HLH proteins.

There is extensive documentation that ld proteins promote cell proliferation and negatively regulate differentiation. High levels of Id gene expression have also been observed in tumor cell lines derived from different tissues
$[1,2]$. In accordance with this, one of the members of this gene family (Id-1) has been shown to promote proliferation and to inhibit functional differentiation of mouse mammary epithelial cells (SCp2 cells), maintained in cell culture [3].

The normal mammary gland is composed of several cell types, but it is the luminal epithelial cells lining the inside of the ducts and the lobules that are primarily targeted for proliferation, differentiation and carcinogenesis. Therefore, to assess the precise significance of any regulatory factor in mammary biology and its significance to carcinogenesis, it is essential to examine its cellular localization in vivo. This is particularly important in the case of ubiquitously expressed proteins, such as Ids. Accordingly, in the present study we examined the in situ localization of Id-1 
in normal mammary glands, and we report that Id-1 is not expressed in the luminal epithelial cells.

\section{Materials and methods}

The source of mammary tissues, FVB and BALB/c strains of mice, used for developmental studies were as follows: pubertal ( 6 weeks old), adult nulliparous (12 weeks old), early pregnant ( 6 days gestation), lactating (day 7 , postpartum), and postlactational involution ( 3 days after pup removal). Id-1 null mutant mice (129Sv/C57BL) have been described previously [4]. For these null mutant mice, the corresponding strain of wild type mice was used as a control. The mice were housed and cared for in accordance with the National Institutes of Health guide to humane use of animals in research.

For immunoblot analyses, tissues were frozen in liquid nitrogen and stored at $-70^{\circ} \mathrm{C}$ until use. For immunohistochemical analyses, mammary glands were fixed in 4.7\% buffered formalin, dehydrated, embedded in paraffin and cut into $5 \mu \mathrm{m}$ thick sections. Tissue sections from paraffinembedded normal human breast were kindly provided by Dr Paul Yaswen.

\section{Source of anti-Id-1 antibody}

An anti-ld-1 rabbit polyclonal antibody (C-20) and the peptide used for the generation of the antibody were purchased from Santa Cruz Biotechnology (Santa Cruz, CA, USA).

\section{Immunoblot analyses}

Protein extracts were prepared from mammary tissues by homogenization in lysis buffer $(50 \mathrm{mM}$ Tris- $\mathrm{HCl}$ [pH 8.0], $125 \mathrm{mM} \mathrm{NaCl}, 1 \mathrm{mM}$ sodium fluoride, $1 \mathrm{mM}$ sodium orthovanadate, $10 \mathrm{mM}$ sodium pyrophosphate and $1 \mathrm{mM}$ phenylmethylsulfonyl fluoride) containing the protease inhibitors leupeptin, pepstatin and aprotinin, each at a final concentration of $1 \mu \mathrm{g} / \mathrm{ml}$. The homogenates were sonicated, centrifuged at $110 \mathrm{~g}$, and the pellets were discarded. Protein concentrations in the supernatants (lysates) were determined by DC protein assay (BioRad, Hercules, CA, USA). Aliquots of mammary gland lysates equivalent to $40 \mu \mathrm{g}$ protein were subjected to electrophoresis through 10-20\% gradient gels and transferred to nitrocellulose membranes. The membranes were blocked with 10\% nonfat powdered milk prior to treatment with the primary antibody. The blots were subsequently washed and treated with appropriate secondary antibodies. The resulting antigen-antibody complexes were detected by the ECL system (Amersham Pharmacia biotech, Chalfont, UK).

\section{Analysis for in situ localization of Id-1}

For immunohistochemistry, tissue sections were deparaffinized, rehydrated, and soaked in antigen unmasking were then heated in the microwave oven for $21 \mathrm{~min}$ to reveal antigens. The sections were incubated with Immuno Pure Peroxidase Suppressor (Pierce, Rockford, IL, USA) to quench the endogenous peroxidase for 1 hour. The Biotin/Avidin blocking kit (Vector) was then used to block the nonspecific background. The antigen-antibody complexes were identified using the Universal DAKO LSAB2labeled streptavidin-biotin peroxidase kit (DAKO, Carpinteria, CA, USA). The sections were counterstained with Mayer's hematoxylin solution (DAKO).

\section{Results \\ Validation of the antibody}

The Id-1 antibody used in the present studies (C-20) was the same as that used previously in immunoblot analyses for demonstrating Id-1 expression in various tissue/cell extracts, including mammary cells [3,5-8]. As shown in Figure 1, in immunoblot analyses of whole mammary gland extracts of wild type mice (lanes 1 and 3 corresponding to 129Sv/C57BL and FVB, respectively), two immunoreactive bands were detected in the region of $15-20 \mathrm{kDa}$. Among these, the top band was absent in extracts prepared from mammary glands of Id-1 null mutant mice (Fig. 1, lane 2), indicating that it corresponded to Id-1. In contrast, in extracts of wild type mouse testis, as reported previously [5], a single immunoreactive band was detected (Fig. 1, lane 4) and this corresponded to the top band in mammary extracts. Similarly, only the top band was detected in extracts of wild type mouse uterus and intestine (Fig. 1, lanes 5 and 6, respectively). Both the top and the bottom bands, detected in mammary extracts of wild type mice, were absent when blots were incubated with Id- 1 antibody in the presence of the peptide used for generation of the antibody (Fig. 1, lane 7). These bands were also absent with the deletion of the primary antibody (data not shown). These observations thus confirmed that the C-20 antibody was capable of detecting ld- 1 , and also demonstrated its presence in whole mammary gland extracts.

The C-20 antibody is known to detect ld- 1 in paraffinembedded tissue sections and has been used successfully for the analyses of Id-1 in sertoli cells of testis by immunohistochemistry [5]. Nevertheless, we verified the ability of the C-20 antibody to detect Id-1, with fidelity, in immunohistochemical analyses. To achieve this we analyzed the vasculature of the developing brain, since Id-1 gene expression has been demonstrated previously in this tissue, by in situ hybridization [9]. Immunoreactivity was detected in the nuclei of the vasculature of the developing brain, as shown in Figure $2 A, B$, and the pattern of immunostaining was similar to that observed for ld-1 gene expression.

\section{Id-1 is not detectable in luminal epithelial cells of mammary glands}

We next examined the in situ localization of Id-1 in mammary glands. As shown in Figure $3 \mathrm{~A}$, immunoreactiv- 


\section{Figure 1}

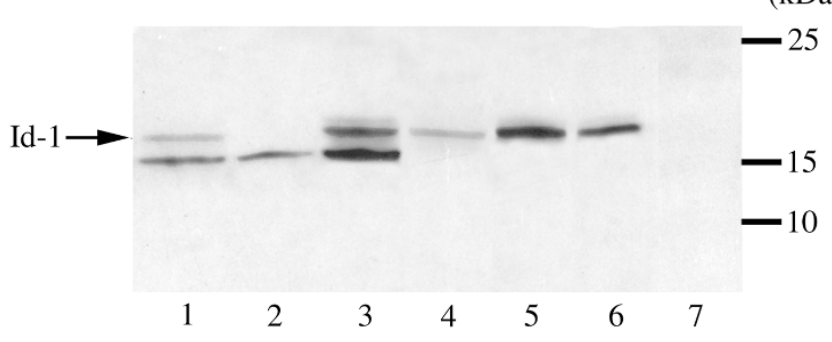

Immunoblot analyses for Id-1 in various mouse tissues. Lysates were prepared from various mouse tissues and analyzed for ld-1, using C-20 antibody, as described in the text. The positions of the molecular weight standards $(\mathrm{kDa})$ are shown on the right. Lanes 1 and 3 , wild type mammary glands (129Sv/C57BL and FVB, respectively); lane 2, mammary glands from Id-1 null mutant mouse; lanes 4-6, testis, uterus and intestine from wild type mice, respectively; lane 7, mammary gland extract of wild type mice in which treatment with the primary antibody was performed in the presence of the blocking peptide.

\section{Figure 2}

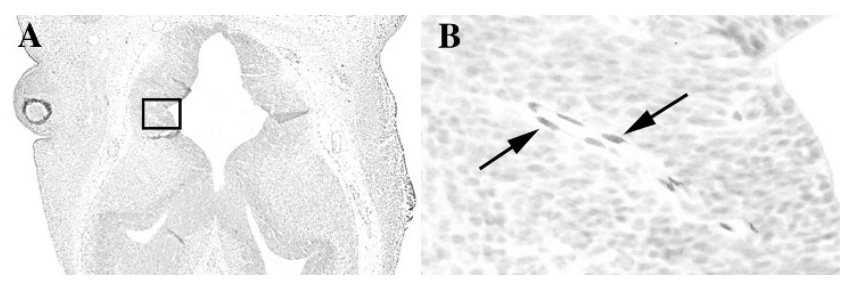

Immunohistochemical analyses for Id-1. (A), (B) The immunoreactivity in the vasculature (arrows) of the developing brain (inset, B) at day 12.5 of gestation. Original magnification, (A) $50 \times$, (B) $400 \times$. ity was not detected either in the luminal epithelial cells or in the stroma of mammary glands of adult nulliparous females. Immunoreactivity was detected, however, in the cytoplasm of myoepithelial cells, and this was completely abolished in the presence of the blocking peptide used for the generation of Id-1 (C-20) antibody (Fig. 3B). Nevertheless, the staining observed in the myoepithelial cells did not appear to be specific to Id-1 since it was also present in mammary myoepithelial cells of Id-1 null mutant mice (compare Fig. 3D and 3E). Similar to mouse mammary glands, Id-1 immunoreactivity was also not observed in luminal epithelial cells of normal human mammary glands, but was detected in the myoepithelial cells (Fig. 3C).

The fact that Id-1 could be detected in mouse mammary tissue extracts by immunoblot analyses but was undetectable in mammary cells by immunohistochemical analyses led us to consider that the Id-1 detected in tissue extracts might have been derived from nonmammary cells. A potential source was vascular endothelial cells since Id-1 is also expressed in the blood vessels outside the central nervous system [9]. Immunoreactivity was detected in the vascular endothelial cells in mammary glands of wild type adult mice (Fig. 3F), but not in the cells of Id-1 null mutant mice (Fig. 3E, inset).

It is well known that mammary epithelial cells of adult nonpregnant females, for the most part, are mitotically quiescent and proliferate extensively only with the onset of pregnancy [10]. Therefore, to examine whether the lack of expression of Id-1 in the luminal epithelial cells was related to its quiescent state, we also analyzed the tissues from early pregnant females. The patterns of immunostaining in mammary glands did not change either during pregnancy

\section{Figure 3}

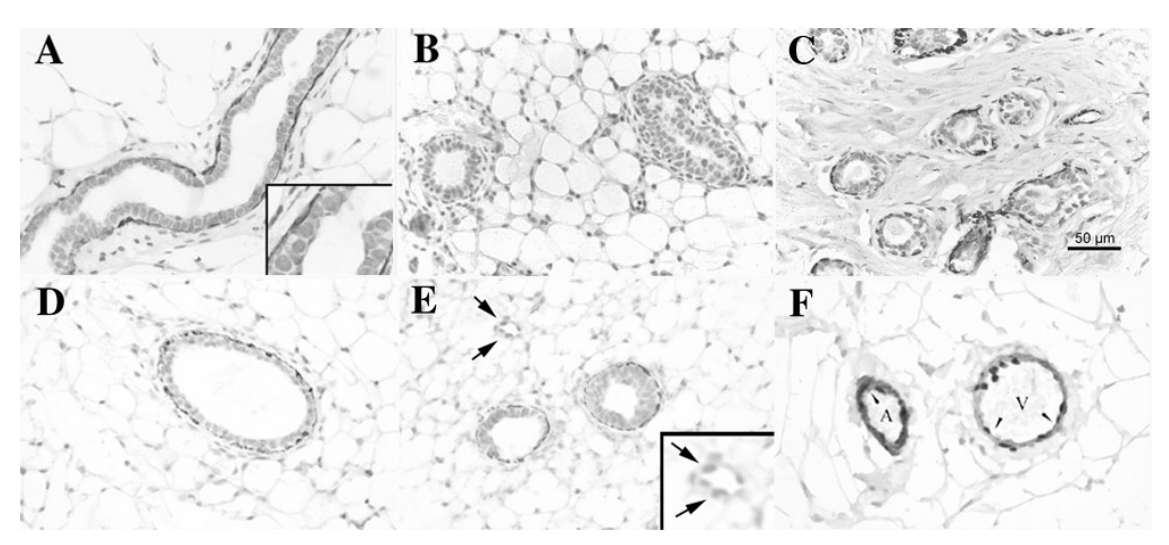

Immunohistochemical analyses for Id-1 in mammary glands. All tissue sections were treated with the primary antibody (except (B), which was incubated with both the primary antibody and the blocking peptide) and processed as described in the text. (A), (B) Adult nulliparous wild type mice (FVB), (C) normal human mammary gland, (D) adult nulliparous wild type mice (129Sv, C57BL/6) corresponding to the strain of Id-1 null mutant mice, (E) arrows showing the vascular endothelial cells, and $(F)$ vascular endothelial cells (arrows) in an artery $(A)$ and a vein $(V)$ in mammary glands of wild type mice. Original magnification: $(A)-(E) 400 \times$. Insets of $(A)$ and $(E)$, higher magnification $(630 \times)$. 
Figure 4

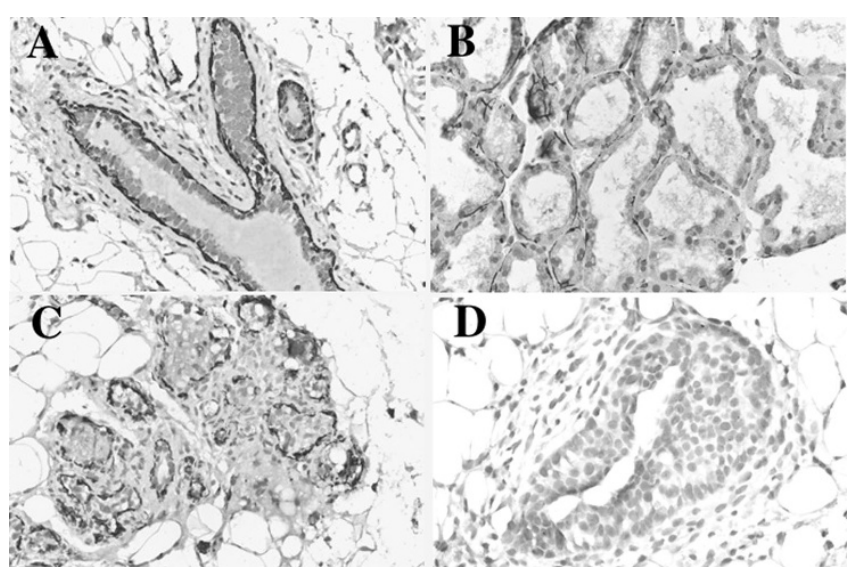

Immunohistochemical analyses for Id-1 in mouse mammary glands during various phases of development. Mammary glands from (A) early pregnant, (B) lactating, (C) lactational involuting and (D) pubertal mice were analyzed for the presence of Id-1 as described in the text. Note that, in all the mammary ducts, immunoreactivity is present only in myoepithelial cells and not in the luminal cells. Immunoreactivity is also not detected in the terminal end bud of the pubertal mouse. Original magnification, $400 \times$.

(Fig. 4A), during lactation (Fig. 4B) or during postlactational involution (Fig.4C). As such, immunoreactivity was still confined to the cytoplasm of the myoepithelial cells and was not detected in luminal epithelial cells. Similar results were also obtained with tissues isolated from the Balb/c strain of mice (data not shown), the strain from which SCp2 cells had been derived [11]. In addition to pregnancy, mammary glands also proliferate extensively at the onset of puberty and, in these glands, the site of intense mitotic activity resides in specialized structures called the terminal end buds [12]. In terminal end buds of pubertal females, immunoreactivity was not detected either in the body cells or the cap cells (Fig. 4D) present at the tips.

\section{Discussion}

In the present report, we have demonstrated that Id-1 is not detectable in the luminal epithelial cells of both mouse and human mammary glands. Our inability to detect Id-1 in these cells is not related to the source of the antibody or techniques since it was possible to detect ld-1 in vascular endothelial cells by immunohistochemistry and via immunoblot analyses of several mouse tissue extracts, including mammary glands. The fact that ld-1 is detected in mammary tissue extracts by immunoblot analyses but not by immunohistochemical analyses therefore indicates that ld-1 detected in whole tissue extracts is derived from nonmammary cells. This argument is supported by our demonstration that Id-1 is expressed in vascular endothelial cells of mammary glands. Furthermore, in contrast to Id-1, we can detect Id-2 in the luminal epithelial cells of mouse unpublished observation) in which Id-2 gene expression has been demonstrated by in situ hybridization [13]. Based on all these observations, we conclude that Id-1 is not expressed in the luminal epithelial cells of mammary glands.

Our studies also demonstrate clearly that in mice, regardless of the strain or the developmental stage, ld-1 is not detectable in the luminal epithelial cells; this included both puberty and early pregnancy, when mammary glands undergo extensive proliferation. Accordingly, our present observations do not support the previous suggestion that, in mammary epithelial cells, $\mathbf{l d}-1$ is a positive and a negative regulator of proliferation and of differentiation, respectively [3]. In turn, the observations also emphasize that the postulated roles for Id-1, using various cell culture models, may not be applicable to all cell types, particularly in vivo [2]. This is exemplified by the fact that much of the information demonstrating various regulatory roles for Id proteins have used fibroblasts and, as shown here, mammary fibroblasts do not express ld-1.

Finally, the detection of 'non-ld-1' immunoreactivity in immunoblot analyses and in myoepithelial cells, with immunohistochemistry, requires comment. It is clearly not due to an abundant nonspecific protein since the immunoreactivity associated with the myoepithelial cells is quite discreet. Indeed, the immunoreactivity associated with the myoepithelial cells is completely abolished by the blocking peptide (Fig. 2). It is also not due to Id-2 or ld-3, since this antibody is specific to an epitope in the carboxy terminus of Id-1 and has no crossreactivity with these proteins $[6,8]$. Furthermore, the nonspecific band detected in immunoblots appears to have some specificity for mammary glands since, to date, we have not detected this band in other mouse tissue extracts. Also, the nonspecific band is eliminated upon exposure to the blocking peptide.

Myoepithelial cells express a number of proteins but several of these are also expressed by luminal epithelial cells [14]. As such, very few proteins are expressed exclusively in the myoepithelial cells, the most prominent one being alpha smooth muscle actin [15]. It is, however, unlikely, that the 'non-ld-1' immunoreactivity associated with myoepithelial cells is alpha smooth muscle actin since it has minimal homology to $I d-1$ and, in particular, to the peptide used for the generation of the antibody. It is therefore most probably due to some other smooth muscle cellspecific protein capable of recognizing the epitope on the Id-1 (C-20) antibody. Identification of this protein can thus lead to establishing another myoepithelial cell-specific marker, which in turn can contribute to our current understanding of the biology of mammary myoepithelial cells.

\section{Conclusion}

Id-1 detected in whole mammary gland extracts is not derived from luminal epithelial cells. 


\section{Competing interests}

None declared.

\section{Acknowledgements}

These studies were supported by a grant from NIH (CA 66541) to GS, a grant from State of California Breast Cancer Research program (5Jb0014) to RDC, and a grant from Breast Cancer Research Foundation to RB. NU was supported by institution training grant DAMD17-00-10224. PdC was supported by a fellowship from the Italian American Cancer Foundation.

\section{References}

1. Israel MA, Hernandez MC, Florio M, Andres-Barquin PJ, Mantani $\mathrm{A}$, Carter $\mathrm{JH}$, Julin CM: Id gene expression as a key mediator of tumor cell biology. Cancer Res 1999, 59:1726s-1730s.

2. Norton JD: ID helix-loop-helix proteins in cell growth, differentiation and tumorigenesis. J Cell Sci 2000, 113:3897-3905.

3. Desprez PY, Hara E, Bissell MJ, Campisi J: Suppression of mammary epithelial cell differentiation by the helix-loop-helix protein Id-1. Mol Cell Biol 1995, 15:33983404.

4. Yan W, Young AZ, Soares VC, Kelley R, Benezra R, Zhuang Y: High incidence of T-cell tumors in E2A-null mice and E2A/ld1 double-knockout mice. Mol Cell Biol 1997, 17:7317-7327.

5. Sablitzky F, Moore A, Bromley M, Deed RW, Newton JS, Norton JD: Stage- and subcellular-specific expression of Id proteins in male germ and Sertoli cells implicates distinctive regulatory roles for Id proteins during meiosis, spermatogenesis, and Sertoli cell function. Cell Growth Differ 1998, 9:10151024.

6. Lin $\mathrm{CQ}$, Singh J, Murata $\mathrm{K}$, Itahana $\mathrm{Y}$, Parrinello $\mathrm{S}$, Liang $\mathrm{SH}$, Gillett CE, Campisi J, Desprez PY: A role for Id-1 in the aggressive phenotype and steroid hormone response of human breast cancer cells. Cancer Res 2000, 60:1332-1340.

7. Wilson JW, Deed RW, Inoue T, Balzi M, Becciolini A, Faraoni P, Potten CS, Norton JD: Expression of Id helix-loop-helix proteins in colorectal adenocarcinoma correlates with p53 expression and mitotic index. Cancer Res 2001, 61:88038810.

8. Parrinello S, Lin CQ, Murata K, Itahana $\mathrm{Y}$, Singh J, Krtolica A, Campisi J, Desprez PY: Id-1, ITF-2, and Id-2 comprise a network of helix-loop-helix proteins that regulate mammary epithelial cell proliferation, differentiation, and apoptosis. J Biol Chem 2001, 276:39213-39219.

9. Lyden D, Young AZ, Zagzag D, Yan W, Gerald W, O'Reilly R, Bader BL, Hynes RO, Zhuang Y, Manova K, Benezra R: Id1 and Id3 are required for neurogenesis, angiogenesis and vascularization of tumour xenografts. Nature 1999, 401:670-677.

10. Shyamala G: Progesterone signaling and mammary gland morphogenesis. J Mammary Gland Biol Neoplasia 1999, 4:89104.

11. Desprez $P$, Roskelley $C$, Campisi J, Bissell MJ: Isolation of functional cell lines from a mouse mammary epithelial cell strain: the importance of basement membrane and cell-cell interaction. Mol Cell Differ 1993, 1:99-110.

12. Williams JM, Daniel CW: Mammary ductal elongation: differentiation of myoepithelium and basal lamina during branching morphogenesis. Dev Biol 1983 97:274-290.

13. Mori S, Nishikawa SI, Yokota Y: Lactation defect in mice lacking the helix-loop-helix inhibitor Id2. EMBO J 2000, 19:57725781.

14. Page MJ, Amess B, Townsend RR, Parekh R, Herath A, Brusten L, Zvelebil MJ, Stein RC, Waterfield MD, Davies SC, O'Hare MJ: Proteomic definition of normal human luminal and myoepithelial breast cells purified from reduction mammoplasties. Proc Natl Acad USA 1999, 96:12589-12594.

15. Lakhani SR, O'Hare MJ: The mammary myoepithelial cellCinderella or ugly sister? Breast Cancer Res 2001, 3:1-4.

\section{Correspondence}

Dr Gopalan Shyamala, Lawrence Berkeley National Laboratory, One Cyclotron Road, Building 74, University of California, Berkeley, CA 94720, USA. Tel: +1 510486 6675; e-mail: Shyamala_Harris@lbl.gov 